\title{
Normalization factors for magnetic relaxation of small-particle systems in a nonzero magnetic field
}

\author{
Ll. Balcells \\ Institut de Ciencia de Materials de Barcelona-CSIC, Campus de la UAB, 08193 Bellaterra, Spain \\ O. Iglesias* and A. Labarta \\ Departament de Física Fonamental, Facultat de Física, Universitat de Barcelona, Diagonal 647, 08028 Barcelona, Spain
}

(Received 1 August 1996; revised manuscript received 11 November 1996)

\begin{abstract}
We critically discuss relaxation experiments in magnetic systems that can be characterized in terms of an energy barrier distribution, showing that proper normalization of the relaxation data is needed whenever curves corresponding to different temperatures are to be compared. We show how these normalization factors can be obtained from experimental data by using the $T \ln \left(t / \tau_{0}\right)$ scaling method without making any assumptions about the nature of the energy barrier distribution. The validity of the procedure is tested using a ferrofluid of $\mathrm{Fe}_{3} \mathrm{O}_{4}$ particles. [S0163-1829(97)04113-1]
\end{abstract}

\section{INTRODUCTION}

The study of the relaxation of magnetic systems provides a way to obtain information about different properties that cannot be so easily achieved by other methods. Most of the works in this field are based on the logarithmic or critical volume approximation. ${ }^{1}$ To characterize the time dependence of the magnetization they analyze the relaxation rate, also called magnetic viscosity, $S$, as a function of the external parameters. When plotted as a function of the magnetic field it is possible to study the variation of the energy barriers with the field, ${ }^{2}$ the interaction effects among the magnetic entities, ${ }^{3,4}$ and the switching field distribution ${ }^{5,6}$ among other magnetic properties. When plotted as a function of the temperature for a given field it gives information about the reversal mechanisms in films and small-particle systems ${ }^{7}$ and it has been proposed as a way to test the possibility of observing macroscopic quantum tunneling (MQT) effects at low enough temperatures. ${ }^{8}$ It is obvious that in this case the initial and final states of the relaxing magnetization are, in general, temperature dependent. Therefore, this dependence must be corrected in $S$ data in order to compare values obtained at different temperatures. If this correction is not taken into account, this trivial contribution to the thermal dependence of $S$ can obliterate the real $S(T)$ behavior arising from the relevant physical mechanism and it can even give rise to misleading interpretations.

Moreover, in some cases, $T \ln \left(t / \tau_{0}\right)$ scaling has been used $^{9,10}$ to confirm the existence of MQT by adducing that the fail of scaling of low-temperature relaxation curves could be the signature of nonthermal mechanisms. As we will show later this lack of scaling could be only a consequence of a nonproper normalization of the data.

In most particle systems in the blocked regime and, due to the long-time decay towards the equilibrium state, it is very difficult to obtain a direct experimental determination of the equilibrium magnetization when a magnetic field is applied [note that, in general, the field-cooled (FC) state is not a true equilibrium state]. It is the purpose of this article to give a detailed account of the normalization procedure of the relaxation curves based on the so-called $T \ln \left(t / \tau_{0}\right)$ scaling method $^{11,12}$ from which an indirect determination of the thermal dependence of the equilibrium magnetization (final state) can be obtained. We also discuss the consequences of this normalization procedure on the analysis of the $S(T)$ behavior. The proposed method is illustrated by using experimental data from a ferrofluid composed of $\mathrm{Fe}_{3} \mathrm{O}_{4}$ small particles.

\section{NORMALIZATION FACTORS FOR RELAXATION CURVES}

In the study of time-dependent processes in small-particle systems two kinds of experiments (which will be called type $\mathrm{A}$ and B) can be distinguished according to what is the final equilibrium state of the system. In type-A experiments the system evolves towards a demagnetized state in zero applied field after a previous cooling in the presence of a field $H$ (FC process) and the variation of the thermoremanent magnetization (TRM) is measured. If $t$ is the time elapsed after the field was reduced to zero, then, in the critical volume approximation, ${ }^{6}$

$$
\begin{aligned}
M_{\mathrm{TRM}}(T, H, t) & =\int_{E_{c}(T, t)}^{\infty} d E M_{\mathrm{FC}}(E) f(E) \\
& \simeq M_{\mathrm{FC}}(T, H) \int_{E_{c}(T, t)}^{\infty} d E f(E),
\end{aligned}
$$

where $E_{c}$ is the critical energy barrier $E_{c}(T, t)=T \ln \left(t / \tau_{0}\right)$ which indicates the onset of superparamagnetic (SP) behavior.

In type-B experiments a zero-field-cooled (ZFC) sample increases its magnetization in a magnetic field $H$ and the variation of the isothermal remanent magnetization (IRM) is measured. In the critical volume approximation the time dependence of the magnetization is given in this case by ${ }^{6}$ 


$$
\begin{aligned}
M_{\mathrm{IRM}}(T, H, t) & =\int_{0}^{E_{c}(T, t, H)} d E M_{\mathrm{eq}}(E) f(E) \\
& \simeq M_{\mathrm{eq}}(T, H) \int_{0}^{E_{c}(T, t, H)} d E f(E) .
\end{aligned}
$$

In order to compare relaxation curves measured at different temperatures it is necessary to remove the thermal dependence of the initial and final states of the magnetization. It is clear from Eqs. (1) and (2) that, due to the fact that in both expressions the integrals are bounded between 0 and 1, this can be simply achieved by dividing magnetization data by a normalization factor which in type-A experiments is $M_{\mathrm{FC}}(T, H)$ and in type-B experiments is $M_{\mathrm{eq}}(T, H)$.

In type-A experiments, the normalization factor $M_{\mathrm{FC}}(T)$ in Eq. (1) comes from the contribution of the blocked particles to the initial magnetization or, in other words, from the irreversible component of the FC magnetization at the temperature $T$. In many small-particle systems at low $T$ this quantity can be considered as a constant in the range of temperatures usually studied. ${ }^{3}$ In fact, when one represents the relaxation data as a function of the scaling variable $T \ln \left(t / \tau_{0}\right)$ all the curves recorded at different temperatures usually superimpose onto a unique master curve without any normalization factor. ${ }^{12}$

In contrast, in type-B experiments the magnetic field is not zero and SP particles have a temperature-dependent contribution to the magnetization while blocked particles are randomly oriented, giving no net contribution to the magnetization in the field direction. Now the reversible component of the ZFC magnetization gives the main contribution to the normalization factor $M_{\mathrm{eq}}(T)$. In many cases, at low fields, $M_{\text {eq }}(T)$ follows a Curie-Weiss law and cannot be considered as a constant. The need of this temperature-dependent normalization factor is clearly manifested as a vertical shift of the curves when data are represented in a $T \ln \left(t / \tau_{0}\right)$ scaling plot.

\section{EXPERIMENTAL RESULTS}

The studied sample is a ferrofluid composed of $\mathrm{Fe}_{3} \mathrm{O}_{4}$ small particles with volume packing fraction $\epsilon=0.01$ which was obtained from chemical deposition of $\mathrm{Fe}^{2+}$ and $\mathrm{Fe}^{3+}$ sulfides and dispersed in a hydrocarbon oil. The sample analyzed by transmission electron microscopy (TEM) was prepared by wetting a carbon film mounted on a $\mathrm{Au}$ grid with the ferrofluid and subsequently drying it with air.

In Fig. 1 an electron micrograph of the magnetic particles is shown. Taking a sampling of 200 particles and considering them spherical, the particle size distribution has been fitted to a logarithmic-normal function with $\sigma=0.24$ and mean particle diameter of about $4 \mathrm{~nm}$ (see inset of Fig. 1).

\section{A. Magnetic characterization}

The magnetic study was performed with a commercial superconducting quantum interference device (SQUID) magnetometer. The highest applied field was $55 \mathrm{kOe}$ and the lowest temperature was $1.8 \mathrm{~K}$.

Figure 2 shows the thermal dependence of the magnetization in an applied magnetic field of 10 Oe following a
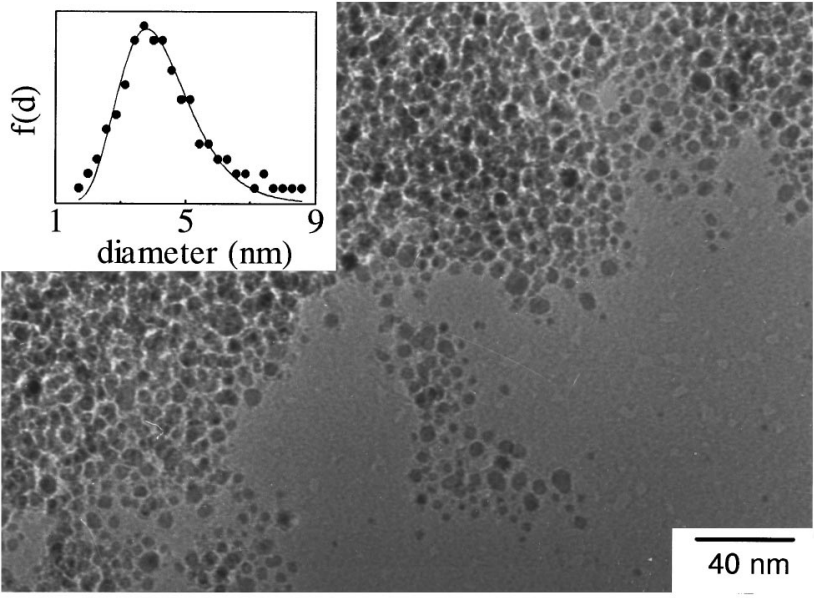

FIG. 1. Electron micrography of the sample obtained by TEM. The inset shows the distribution of particle diameters obtained from a sampling of 200 particles. The solid line is a logarithmic-normal function with $\sigma=0.24$ and mean particle diameter of about $4 \mathrm{~nm}$.

ZFC-FC process. The magnetic behavior displayed is typical of SP particles. Above $T_{\mathrm{irr}}=50 \mathrm{~K}$ the system is in the SP region, in which the magnetization curve is reversible. The ZFC curve develops a maximum at $T_{B}$ which is about $14 \mathrm{~K}$.

To obtain the mean magnetic moment of the particles as a function of the temperature, $\bar{\mu}(T)$, we have fitted the magnetization curves deep in the SP region (within the range 80-200 K) to a distribution of Langevin functions following the procedure described in Ref. 13. By extrapolating these values to $T=0 \mathrm{~K}$ with a $T^{3 / 2}$ law (as expected according to a spin-wave theory for a ferrimagnetic material ${ }^{14}$ ), a mean magnetic moment per particle of $\bar{\mu}=(1320 \pm 20) \mu_{B}$ is obtained. Using $\bar{\mu}$ and the bulk magnetic moment of the magnetite the mean magnetic size of the particles has been found to be $3.5 \mathrm{~nm}$ in diameter.

$T_{B}$ has been measured as a function of the field. By extrapolating these values to $T_{B}=0$ we have estimated the mean anisotropy field (the field at which the mean energy barrier disappears) to be $H_{A} \simeq 5000$ Oe. From $H_{A}$ the mean

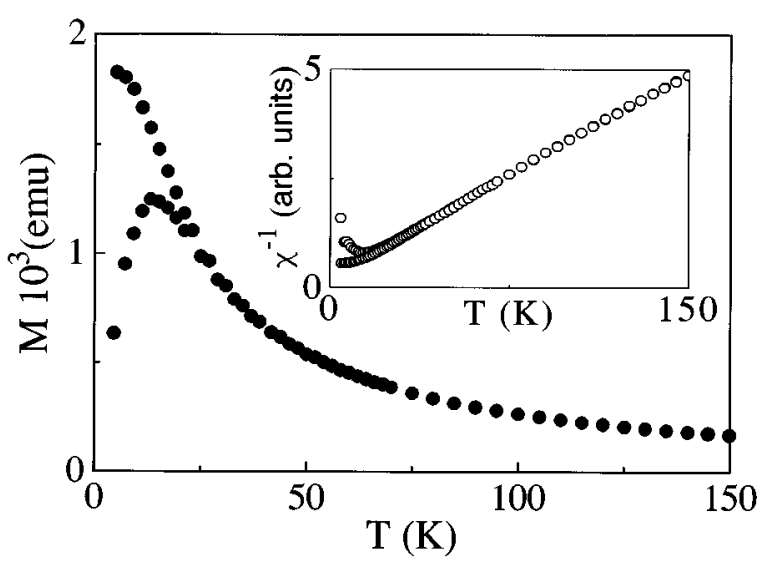

FIG. 2. Temperature dependence of the magnetization of the sample for a ZFC (lower curve) FC (upper curve) process in a magnetic field of 10 Oe. Inset: reciprocal of the ZFC-FC susceptibility corrected to take into account the thermal variation of the mean particle moment. 
anisotropy constant of the particles has been estimated as $K=\bar{\mu} H_{A} / 2 \bar{V}=1.3 \times 10^{5} \mathrm{~J} / \mathrm{m}^{3}$, where $\bar{V}$ is the mean particle volume. This value is close to others found in the literature for similar systems. ${ }^{15-17}$

For the subsequent analysis of the relaxation curves it is also convenient to study the variation of the initial susceptibility with the temperature. For a system of interacting magnetic particles in the SP regime this quantity can be written as $^{18}$

$$
\chi \sim \frac{\bar{\mu}^{2}}{3 k_{B}\left(T-T_{0}\right)},
$$

where $T_{0}$ is due to the existence of interparticle interactions and/or the effect of $f(E)$ (see Ref. 18). The inset in Fig. 2 shows the inverse susceptibility as a function of the temperature as obtained from the ZFC-FC curve, where the thermal variation of the mean moment of the particles has been corrected by using the $T^{3 / 2}$ law obtained before. Above $T_{\text {irr }}$, $1 / \chi$ shows a linear dependence on $T$. Fitting $1 / \chi$ to Eq. (3) we have estimated $T_{0}=-11 \pm 3 \mathrm{~K}$. This value of $T_{0}$ may be mainly attributed to the existence of demagnetizing interparticle interactions taking into account that the volume distribution is not very broad.

\section{B. Normalization factors and $T \ln \left(t / \tau_{0}\right)$ scaling}

The magnetic relaxation curves at different temperatures were recorded with a SQUID magnetometer following the procedure described in Ref. 19. The measurements were started $5 \mathrm{~s}$ after applying the field and were performed during approximately $1000 \mathrm{~s}$ at the lowest temperature and approximately $10000 \mathrm{~s}$ at the highest temperature. The relaxation curves after ZFC the system were measured in the presence of a magnetic field of 10 Oe (type-B experiment) while in those measured in zero field the system was previously FC in 10 Oe (type-A experiment).

In the following we will describe an experimental procedure, based on the $T \ln \left(t / \tau_{0}\right)$ scaling method, ${ }^{12}$ to obtain normalization factors $M_{\mathrm{eq}}(T)$, for relaxation data recorded in the presence of a field since they cannot be directly measured due to the long-time decay of the magnetization.

First, the attempt time $\tau_{0}=3 \times 10^{-11} \mathrm{~s}$ has been evaluated by scaling the relaxation curves in zero field after FC the system at several temperatures following the method previously described in Ref. 12. For this purpose we have considered that the initial magnetization $M_{\mathrm{FC}}(T, H)$ is constant in the temperature range of the experiment, and so no normalization factors are needed to scale the curves. We will assume that the field variation of $\tau_{0}$ is smaller than the error in its determination at low fields, according to Brown's theory. ${ }^{20}$

In the next step, relaxation data recorded in the presence of a field have been plotted as a function of the scaling variable using the value of $\tau_{0}$ previously deduced (see Fig. 3). After this transformation the relaxation curves at different temperatures are separated along the vertical axis by temperature-dependent shifts. Taking into account that the applied field is much lower than $H_{A}$ and therefore the energy barriers have not been significatively affected, this lack of scaling is a clear demonstration that magnetization data must be normalized to achieve an equivalent scaling to that ob-

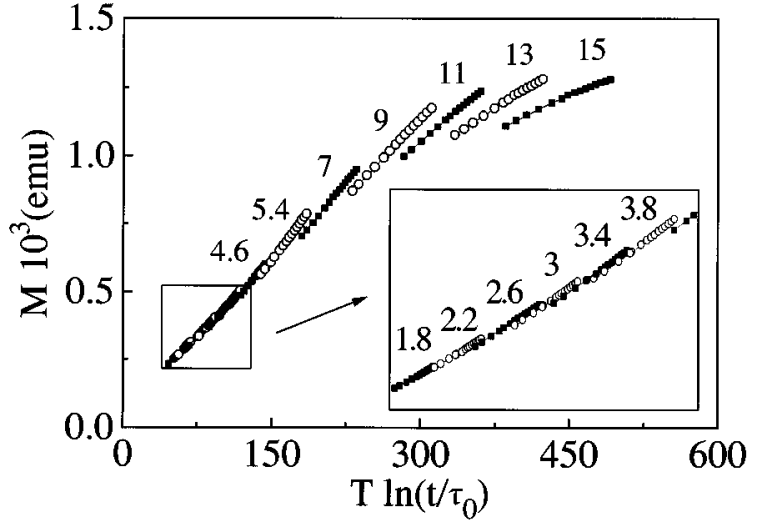

FIG. 3. Relaxation data recorded from 1.8 to $15 \mathrm{~K}$ in the presence of a magnetic field of 10 Oe after ZFC the sample as a function of the $T \ln \left(t / \tau_{0}\right)$ scaling variable. Open and solid symbols correspond alternatively to the temperatures indicated in the figure. Inset: detail of the lowest-temperature region.

tained in zero field. As has been discussed in Sec. II the normalization factors are proportional to $M_{\text {eq }}(T, H)$. If now we assume that $M_{\mathrm{eq}}(T, H)$ are given by $M_{\mathrm{FC}}(T, 10 \mathrm{Oe})$, as suggested by some authors, ${ }^{10}$ no scaling is achieved because FC magnetization does not correspond to the true equilibrium state. Note that $M_{\mathrm{eq}}(T, H)$ cannot be calculated without making any a priori hypothesis about the form of $f(E)$ and the magnetic microstructure of the system.

The normalization factors can be found by referring the different curves to the lowest-temperature one. Once this process has been performed, the relaxation curves collapse onto a unique master curve that is shown in Fig. 4. The values of the normalization factors follow a Curie-Weiss law of the form (3) with $T_{0}=-15 \pm 2 \mathrm{~K}$ (see inset of Fig. 5). The extrapolation of this law superimposes with the susceptibility corresponding to the reversible (SP) region obtained from ZFC-FC measurements (see Fig. 5), reflecting the fact that for long enough observation times all the particles have become SP and demonstrating that the normalization factors are proportional to $M_{\mathrm{eq}}(T, H)$.

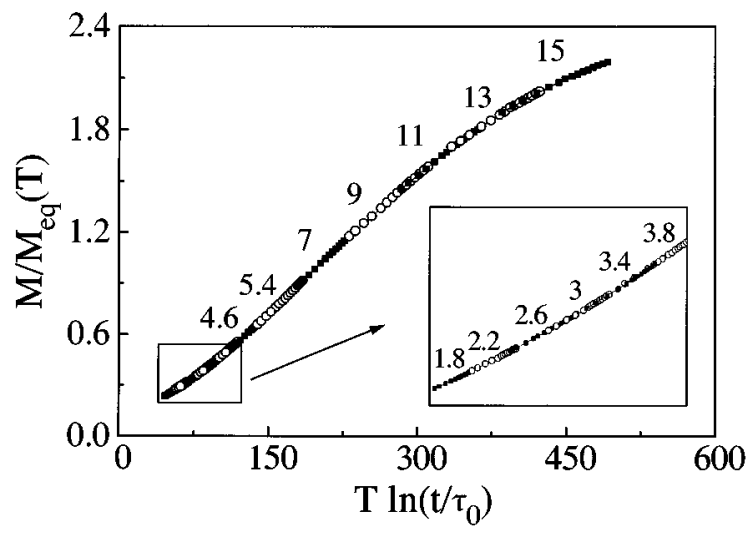

FIG. 4. Scaling plot for the relaxation measurements shown in Fig. 3. Open and solid symbols correspond alternatively to the temperatures indicated in the figure. Inset: detail of the lowest temperature region. 


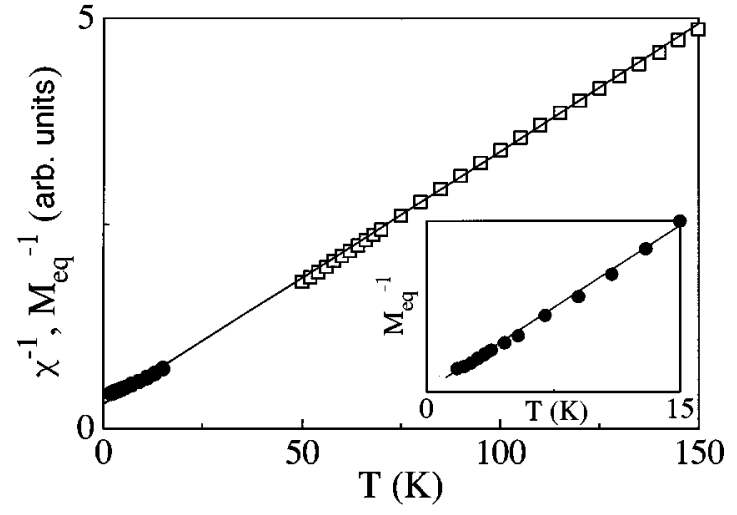

FIG. 5. Reversible region of the reciprocal susceptibility (open squares) and thermal dependence of the inverse of the normalization factors (solid circles) necessary to join the relaxation data of Fig. 3 onto a unique master curve. The solid line is a linear regression of both data. Inset: inverse of the normalization factors as a function of the temperature. Normalization factors have been reduced to the same units of the susceptibility multiplying them by an arbitrary quantity.

\section{Magnetic viscosity and energy barrier distribution}

The classical magnetic viscosity, commonly defined as $S(t, T)=\partial M(t) / \partial(\ln t)$, cannot be directly compared at different temperatures because (1) usually magnetization is not normalized (initial and final states of the relaxation process change as the temperature varies), and (2) if relaxation data have been recorded in a fixed time window, the energy barriers which are relaxing at different temperatures are not the same. Both problems can be circumvented by defining magnetic viscosity as $\bar{S}(t, T)=\partial \bar{M}(t) / \partial\left(T \ln \left(t / \tau_{0}\right)\right)[\bar{M}(t)$ is the normalized magnetization used in the scaling procedure]. By performing the $T \ln \left(t / \tau_{0}\right)$ derivative $\bar{S}$ measures the relaxation rate of the magnetization due only to the energy barriers around $T \ln \left(t / \tau_{0}\right)$. On the other hand, as previously noted in Ref. $21, \bar{S}$ is a magnitude proportional to the energy barrier distribution and therefore it has a direct physical meaning.

These two magnitudes are simply related by

$$
\bar{S}=\frac{S}{M_{\mathrm{FC}} T}
$$

in the case of type-A relaxation experiments and by

$$
\bar{S}=\frac{S}{M_{\mathrm{eq}} T}
$$

for type-B experiments. In the first case and for systems with a certain degree of interaction between particles, $M_{\mathrm{FC}}$ is usually almost temperature independent and both magnitudes differ by a $T^{-1}$ factor. Therefore conclusions from the thermal variation of $S$ obtained from type-A experiments must be carefully derived. Note in particular that if $S$ happens to be temperature independent in a certain range, a result which could be interpreted as a proof of the existence of quantum relaxation phenomena, ${ }^{22}$ this would be a consequence of an energy barrier distribution proportional to $1 / E$ in this range, as the $\bar{S} \sim T^{-1}$ thermal dependence reveals. It is worth noticing that if there is not a certain degree of freezing due to

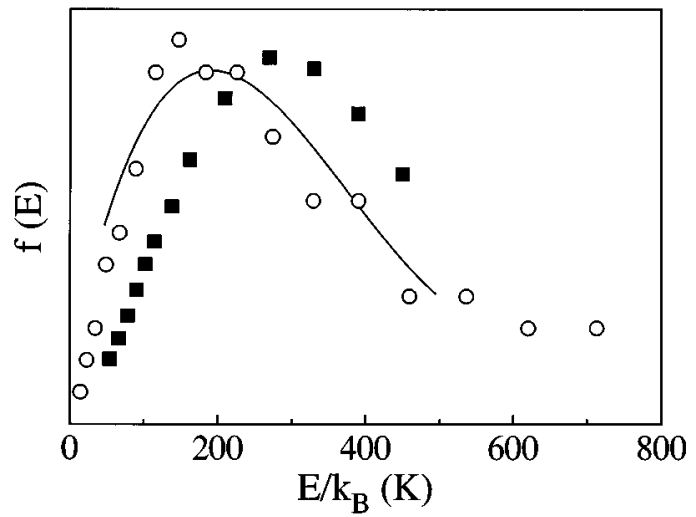

FIG. 6. Effective distribution of energy barriers as obtained from the numerical derivative of the master relaxation curve (solid line). Magnetic viscosity $S(T)$ as obtained from the logarithmic time derivative of the relaxation data at the temperatures indicated in Fig. 4 (solid squares). The energy distribution $f(E)$ obtained from $f(V)$ is also shown for comparison (open circles).

magnetic interactions, $M_{\mathrm{FC}}$ cannot be considered as a constant and its thermal variation must be corrected in $S$. However, in type-B experiments, only if $M_{\text {eq }}(T)$ is inversely proportional to the temperature (Curie law), as is the case for a sample with no or very small interparticle interactions and a narrow distribution of energy barriers, ${ }^{18}$ will both magnitudes nearly coincide (note that this is not the case of the sample studied in this paper) because the thermal variation of $M_{\text {eq }}$ cancels the factor $T$ in Eq. (5).

For the sample studied in this paper $\bar{S}\left(T \ln \left(t / \tau_{0}\right)\right)$ has been obtained by performing the numerical derivative of the master curve of Fig. 4 and has been compared to the viscosity $S(T)$ as obtained from the logarithmic derivative of the relaxation data at each temperature. The results are shown in Fig. 6 together with the energy distribution obtained from electron micrography by expressing the volume distribution in energy units with the help of the value of $K$ previously derived. The coincidence between $\bar{S}$ and $f(E)$ shows the consistence of the normalization used in the scaling procedure for type-B experiments. On the other hand, it is important to note that $S$ does not coincide with $\bar{S}$ because for this sample $M_{\text {eq }}$ is not simply proportional to $T^{-1}$. Only the overall shape of the energy barrier distribution obtained from TEM is reproduced by $S$, but shifted to higher energies. Note also that the extrapolation of the quasilinear low-temperature regime of $S$ intercepts the temperature axis at a nonzero value as has been reported in other systems. ${ }^{23}$ In our case, this result is only a consequence of the lack of normalization and has no physical meaning.

\section{CONCLUSIONS}

We have stressed the importance of proper normalization whenever relaxation curves measured at different temperatures must be compared. In the case of experiments performed in zero field, care must be taken in systems for which $M_{\mathrm{FC}}(T)$ cannot be considered as a constant. When this is the case, non-normalization could give place to a spurious thermal dependence. 
In the case of relaxation experiments performed in an applied magnetic field, there exists a certain controversy in the literature about the nature of the normalization: Either no normalization factors are used at all ${ }^{8,24,25}$ or the FC magnetization value, corresponding to the field at which the experiment is performed, ${ }^{10}$ is used.

In systems for which the $M_{\mathrm{eq}}(T)$ follows a Curie law (noninteracting particles, negligible $T_{0}$ ) the first option happens to be correct by chance as can be easily seen in Eq. (5). In this kind of system the second option is particularly wrong when applied to low-temperature data because the FC magnetization is slightly temperature dependent while the SP magnetization, which is the true equilibrium state at long times, follows a $T^{-1}$ behavior.

In fact, when $T \ln \left(t / \tau_{0}\right)$ scaling is used to evidence quantum relaxation mechanisms through a lack of scaling of relaxation data (recorded in the presence of a field), no conclusions should be extracted without previously having tried to normalize data following the process described in Sec. III B. The sample studied in this paper is an example where a clear failing of the scaling of the non-normalized data does not indicate any nonthermal process $[\bar{S}$ agrees with the energy distribution deduced from $f(V)$; see Fig. 6]. This does not mean that it is always possible to find scaling factors for data corresponding to low $T$ if MQT occurs. If this is the case, even a multiplicative factor is not enough to superimpose relaxation curves obtained at different $T$ ( $T$ is then an irrelevant parameter that would not have to be included in the scaling variable).

In conclusion, we have shown that the $T \ln \left(t / \tau_{0}\right)$ scaling method provides a useful tool to obtain the normalization factors and the energy barrier distribution in both kinds of experiments and even in systems that cannot be considered as an assembly of independent small particles (i.e., multilayered systems, cluster glasses, amorphous alloys, etc.) without making any assumptions about the nature of the magnetic microstructure.

\section{ACKNOWLEDGMENTS}

We are indebted to Professor S.W. Charles for providing the sample studied in this paper. Financial support from both the Spanish CICYT through Grant No. MAT94-1024CO2-02 and the Catalan CIRIT through Grant No. GRQ1012 is acknowledged.
*Author to whom correspondence should be sent. Electronic address: oscar@hermes.ffn.ub.es

${ }^{1}$ C. P. Bean and J. D. Livingstone, J. Appl. Phys. 30, 120S (1959).

${ }^{2}$ B. Barbara, L. C. Sampaio, A. Marchand, O. Kubo, and H. Takeuchi, J. Magn. Magn. Mater. 136, 183 (1994); R. W. Chantrell, ibid. 95, 365 (1991); G. W. D. Spratt, P. R. Bissell, R. W. Chantrell, and E. P. Wohlfarth, ibid. 75, 309 (1988).

${ }^{3}$ K. O'Grady and R. W. Chantrell, in Magnetic Properties of Fine Particles, Proceedings of the International Workshop on Studies of Magnetic Properties of Fine Particles, Rome, edited by J. L. Dormann and D. Fiorani (North-Holland, Amsterdam, 1992), p. 103.

${ }^{4}$ M. García del Muro, X. Batlle, A. Labarta, J. M. González, and M. I. Montero (unpublished).

${ }^{5}$ G. W. D. Spratt, P. R. Bissell, and R. W. Chantrell, IEEE Trans. Magn. 23, 186 (1987); X. Batlle, M. García del Muro, and A. Labarta, Phys. Rev. B 55, 6440 (1997).

${ }^{6}$ K. O'Grady and R. W. Chantrell, in Ref. 3, p. 93.

${ }^{7}$ R. W. Chantrell, A. Lyberatos, M. El-Hilo, and K. O'Grady, J. Appl. Phys. 76, 6407 (1994); L. Folks and R. Street, ibid. 76, 6341 (1994); J. M. González, R. Ramírez, R. Smirnov-Rueda, and J. González, Phys. Rev. B 52, 16034 (1995).

${ }^{8}$ Quantum Tunneling of Magnetization, Proceedings of the NATO Advanced Research Workshop, QTM '94, edited by L. Gunther and B. Barbara (Kluwer Publishing, Dordrecht, The Netherlands, 1995).

${ }^{9}$ J. Tejada et al., in Ref. 8; J. I. Arnaudas et al. in Ref. 8.

${ }^{10}$ E. Vincent, J. Hammann, P. Prené, and E. Tronc, J. Phys. (France) I 4, 273 (1994).
${ }^{11}$ J. J. Préjean and J. Souletie, J. Phys. (France) I 41, 1335 (1980).

${ }^{12}$ A. Labarta, O. Iglesias, Ll. Balcells, and F. Badia, Phys. Rev. B 48, 10240 (1993).

${ }^{13}$ S. Linderoth, L. Balcells, A. Labarta, J. Tejada, P. V. Hendriksen, and S. A. Sethi, J. Magn. Magn. Mater. 124, 269 (1993).

${ }^{14}$ N. W. Ashcroft and N. M. Mermin, Solid State Physics (Holt, Rinehart and Winston, New York, 1979); S. Linderoth, J. Magn. Magn. Mater. 104-107, 167 (1992).

${ }^{15}$ T. Jonsson, J. Mattsson, C. Djurberg, F. A. Khan, P. Nordblad, and P. Svedlindh, Phys. Rev. Lett. 75, 4138 (1995).

${ }^{16}$ W. Luo, S. R. Nagel, T. F. Rosenbaum, and R. E. Rosensweig, Phys. Rev. Lett. 67, 2721 (1991).

${ }^{17}$ C. Johansson, M. Hanson, P. V. Hendriksen, and S. Mørup, J. Magn. Magn. Mater. 122, 125 (1993).

${ }^{18}$ M. El-Hilo, K. O’Grady, and R. W. Chantrell, J. Magn. Magn. Mater. 117, 21 (1992).

${ }^{19}$ A. Labarta, R. Rodríguez, Ll. Balcells, J. Tejada, X. Obradors, and F. J. Berry, Phys. Rev. B 44, 691 (1991).

${ }^{20}$ W. F. Brown, Jr., Phys. Rev. 130, 1677 (1963).

${ }^{21}$ O. Iglesias, F. Badia, A. Labarta, and Ll. Balcells, Z. Phys. B 100, 173 (1996).

${ }^{22}$ See Ref. 8, Chap. 3.

${ }^{23}$ J. Tejada, X. X. Zhang, and C. Ferrater, Z. Phys. B 94, 245 (1994); J. Tejada, X. X. Zhang, and E. M. Chudnovsky, Phys. Rev. B 47, 14977 (1993); J. Tejada, X. X. Zhang, and Ll. Balcells, J. Appl. Phys. 73, 6709 (1993).

${ }^{24}$ P. Perera and M. J. O'Shea, Phys. Rev. B 53, 3381 (1996).

${ }^{25}$ M. M. Ibrahim, S. Darwish, and M. S. Seehra, Phys. Rev. B 51, 2955 (1995). 